\title{
Blockchain for Collaborative Businesses
}

\author{
Augusto R. C. Bedin ${ }^{1} \cdot$ Miriam Capretz ${ }^{1}$ (D) $\cdot$ Syed Mir $^{2}$
}

Published online: 9 October 2020

(C) The Author(s) 2020

\begin{abstract}
Blockchain applications have continuously improved ever since its first debut on cryptocurrency. From then on, its uses have branched out from the financial realm, finding their way into numerous industries such as health, environmental, and governmental. Businesses are starting to take advantage of the intrinsic traits that made blockchain so notorious into their operations, such as security, integrity, and transparency. Blockchain's versatility allows companies to cooperate on a shielded environment with business partners safely. This paper details how permissioned blockchain networks can accommodate collaborative business models securely to provide thriving business alliances. Examples of cooperative business models and business relationship orientation are described here, as well as how they generate value when paired with permissioned blockchain networks - a more business-oriented variety of blockchain. To support this study's endeavors, business use cases are presented to highlight how simple it is to put in place a permissioned blockchain to businesses to achieve tighter bonds with business partners. The use cases contain particular goals that enterprises seek to accomplish by partnering up with other companies, as well as how, with the employment of blockchain, they can attain them.
\end{abstract}

Keywords Blockchain · Permissioned Blockchain · Collaborative Blockchain · Collaborative businesses

\section{Introduction}

Blockchain technology has become more and more widespread over the years after it revolutionized the way that money can be traded, created, and earned. The words Bitcoin, Ethereum and cryptocurrency are so often seen and heard on the news that people are now used to those once unrecognizable terms. The financial applications of blockchain are highly regarded showcases that inevitably causes blockchain to be considered a finance-oriented technology. Blockchain's inherent traits in security and data handling integrity are very enticing for financial applications, but can easily be implemented and taken advantage of by various other business sectors. Any circumstance that would require a high level of trust

Miriam Capretz

mcapretz@uwo.ca

Augusto R. C. Bedin

arodr3@uwo.ca

Syed Mir

mirs@londonhydro.com

1 Department of Electrical and Computer Engineering, Western University, London, ON N6A 5B9, Canada

2 London Hydro, 111 Horton Street, London, ON N6A 4H6, Canada among parties or even a third party intermediate to validate the interaction can benefit from blockchain's trusted environment [22]. Granted blochchain's versatility, it found its way into energy [2], government [25], IoT [11], and even medical [27] industries demonstrating its flexibility in numerous scenarios.

As a distributed ledger technology (DLT), blockchain technology presents itself as a means to tighten bonds between organizations and its customers by providing more data integrity, security, and transparency for its services. Additionally, permissioned blockchains have a unique knack for handling business activities very well. With a more business-centered attitude, permissioned blockchains enable organizations to structure access levels for its members through valid credentials. When this is achieved, an organization's hierarchical system can be installed on the blockchain to do business through a safeguarded medium. Such a feature, which is only available on permissioned blockchains, can be a useful tool for companies to implement social responsibility. Naturally, it is reasonable to contemplate the likelihood of building such an application without adopting blockchain technologies. Although possible, its technical complexity quickly reveals itself a barrier. Blockchain grants, upon majority approval, newcomers a swift integration into the network and so to the data available in it. Security and integrity technicalities are not a matter of contention when dealing with blockchain. 
Nakamoto's first paper on peer-to-peer network [24], which later became known as blockchain, cites how little effort is required not only to set up a blockchain network but also how easily nodes can join or leave the network at will, accepting that the longest chain of blocks in it as proof of everything that happened when they were absent.

Organizations can come together to provide new or better services to their clients, adjusting their business models to accommodate newfound strategies and opportunities. Presented herein are use cases and examples on how differently related companies, whether vertically, horizontally or, specially, diagonally related - which commonly do not have predictable value creation - can join forces towards new business horizons. This paper's focus is to highlight how blockchain can help bridge the trust gap among businesses to consolidate potential partnerships and alliances, shedding light on business opportunities and possibilities. Moreover, this study also supports building a permissioned blockchain environment where organizations can work alongside other organizations to uphold a closer relationship through a candid regime established through smart contracts, direct channels of communications, and transparency due to the nature of distributed ledgers.

The remainder of this paper is organized as follows: Section 2 presents blockchain background information. Related relevant work is discussed in Section 3. Section 4 characterizes business relationships and collaborative models for a more thorough understanding of how blockchain can generate value in different business situations. Section 5 contains examples of use cases of blockchain applications using two different collaborative models and finally, some thoughts and remarks conclude the paper in Section 6.

\section{Background}

To properly understand why blockchain is a secure and safeguarded way to interact with other participants, its comprehension is in order. Terminologies and necessary complementary information on blockchain are reviewed to avoid misinterpretation. Additionally, pertinent business blockchain studies are also discussed here to support and justify this paper's endeavors.

Blockchain technology consists of recording the exchange of information within a network where every user holds a ledger with the records of every movement of every piece of information [13]. Figure 1 illustrates what a sample blockchain network would look like.

This information is exchanged in the form of authorized transactions validated by the network users. An arbitrary number of transactions can then be packed along with an identifier (by default, a hash), establishing a block of information. The blocks are created with the sequential identifier of the previous block and put into a chain of blocks. Once the block is placed into the chain, its inner data become immutable and can no longer be tampered with. Because every piece of information inside the blocks has been previously approved by the majority of users in the network, the data are regarded as legitimate and trustworthy. This process is depicted in Figure 2.

Figure 2 illustrates the primary blockchain modus operandi. The technology is flexible enough to be tailored to handle the desired information that is relevant to its users. In other words, the ledgers on every user's node will contain information that is pertinent to the whole body of users.

- Permissionless Blockchain - The scenario depicted above holds for permissionless, public blockchains. Public blockchain networks allow virtually anyone to join, participate, and access the information within them. Granted, this type of blockchain is presumably large in scale, and therefore to control its anonymous and untrusted nodes, a consensus must be reached either through the approval of a majority of users or through a Proof of Work (PoW) [3]. The PoW is evidence that a user has spent enough computational effort to validate its transaction.

- Permissioned blockchain - Private, permissioned blockchains are generally smaller in scale. In permissioned blockchains, to join, one must hold a valid certificate issued by an accredited entity in the network. This normally means that an administrator node must assign an identity for newcomers so that they are no longer anonymous, and other users/nodes can recognize their actions on the blockchain. Permissioned blockchains act as a semi-trusted setting [3], and because of that, consensus can be reached differently than on permissionless, public blockchains. Consensus on permissioned settings is not as costly to achieve as on permissionless blockchains since nodes are identifiable and trusted within that network. No proof of work is required for the nodes to invoke transactions. The only necessary verification is the validity of the data within a node's transaction.

- Smart contracts - Smart contracts are, in a blockchain frame of reference, code scripts stored within the blockchain [6]. They are triggered autonomously and fundamentally by transactions in a structured way throughout all the nodes in the network, conforming to the data used by the triggering transaction. Figure 3 illustrates how a smart contract execution process works. When invoking a transaction that triggers a smart contract its code will run in every node involved in the transaction, executing the business logic that it was designated for. Smart contracts enable a more fluid work flow by prompting computational procedures on demand, responding involved parties under a structured policy. 
Fig. 1 A blockchain sample network with business partners

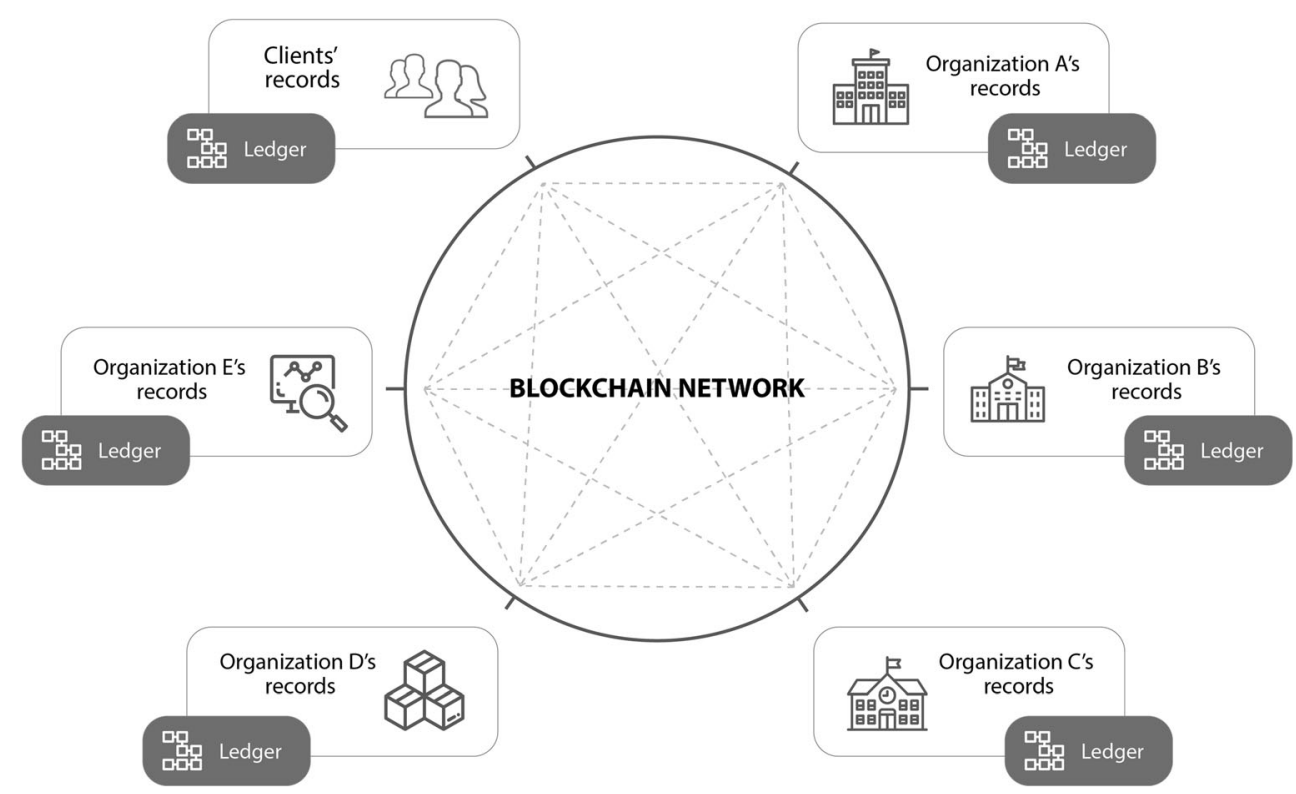

By doing so, they allow a reduced number of trusted intermediaries to be involved in parties' transactions while also minimizing accidental and erroneous transactions.

\section{Related work}

Blockchain for enterprises is significantly on the rise. This is confirmed by the quantity of papers on the topic published in recent years as different applications using blockchain are revealed. Hebert and Di Cerbo [12] outline a methodology for businesses to better reap the benefits of using blockchain in the private sector. It expresses the concerns and advantages of using blockchain while drawing attention to how to mold software architectures to achieve maximum business blockchain efficiency. Various markets, financial and nonfinancial, have become aware of blockchain benefits, and its implementation has spread to seize newfound opportunities [8].

Addressing healthcare, McGhin et al. [20] provide an extensive study and analysis of the blockchain scenario in healthcare. It highlights not only the benefits blockchain delivers in that realm, but also opportunities not yet tackled in that field, along with many challenges to implement the technology into such a complex domain. Liang et al. [18] highlight how permissioned blockchains have presented themselves as a strong ally in dealing with healthcare issues, given their enhanced data security and integrity. Due to the sensitivity and privacy requirements of health data, blockchain has become a powerful tool for safely collaborating and sharing data in this category.

In terms of financial and business data, Chua et al. [7] have presented an example of how permissioned blockchains, specifically Hyperledger Fabric, can serve as a foundation for business activities. It explains how a group of closely collaborative enterprises can gain quick access to data on a distributed ledger network such as a blockchain. In addition, the study stresses how permissioned blockchains provide access control over their data to enable interoperability among the network's participants. Lev-Ari et al. [16] focus on building more trust among the blockchain's participants by gearing Hyperledger's consensus protocol into a more fitting one for financial instituions, where selfish behaviour is more prominently present when trying to achieve utmost profit.

For government purposes, Allessie et al. [1] present a study that shows how blockchain technology can reduce bureaucracy, increase the efficiency of administrative processes and increase the level of trust in public recordkeeping, even though major breakthroughs regarding the usage of blockchain for government have not occurred. Kirillov et al. [14] makes a case for Hyperledger Fabric for e-voting. They
Fig. 2 Blockchain: each block contains an identifier hash pointing to the previous block and a package of transactions that have taken place in the network [6]

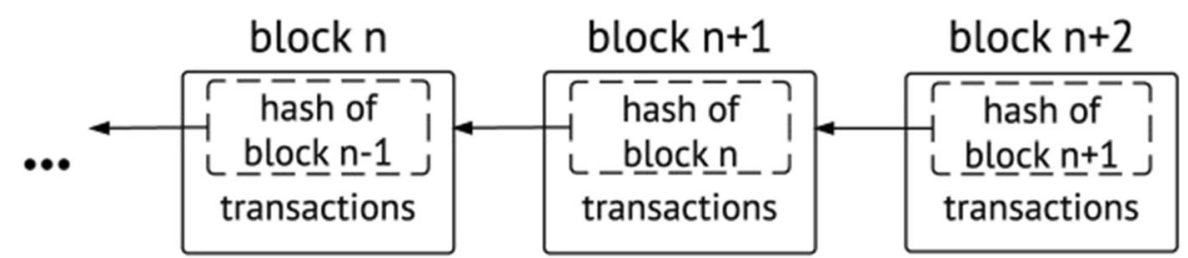



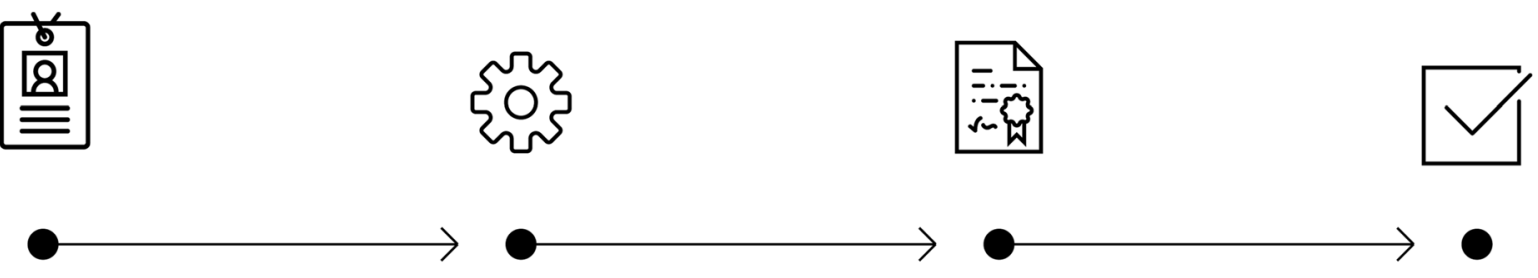

\section{Request transaction}

\section{Transaction triggers smart contract execution}

Smart contracted executed based on pre-agreed terms
Transaction is commited

Fig. 3 How smart contracts are triggered and executed on a valid transaction

propose an e-voting blockchain model to increase trust among network participants. Again, the handling of sensitive data such as government information demands a secure and reliable environment, one that blockchain can easily provide.

The examples portrayed above are just a few examples of environments where a permissioned blockchain could be helpful. As stated previously, social responsibility-focussed blockchain applications are still scarce. Use of a blockchain in support of a social cause was examined in Liu et al. [19], where a blockchain was used to store carbon footprint emissions in Taiwan. The immutability and irreversibility of blockchain data provide a reliable window to check a company's carbon emissions, encouraging more compliant behavior towards environmental regulations and hence towards society overall.

As blockchain applications mature, more complex scenarios can be tackled. On such trend, Li [17] focuses on a philanthropic logistics platform that takes advantage of blockchain's high-profile transparency and credibility. Using Ethereum, the study focused on implementing an application geared towards social welfare maximization, a pertinent example of a social responsibility targeted blockchain.

Blockchain geared towards social responsibility is continuously increasing its popularity given social awareness growth in society as general. Mukkamala et al.'s study [23] outlines several opportunities and applications for blockchain to enhance social business models in pursuit of maximizing its societal impacts as well as their profits. United Nations Research Institute for Social Development's (UNRISD) working paper [26] highlights blockchain's potential for creating cooperation at scale with cooperative structures for financial inclusion. Kouhizadeh and Sarkis study [15] discusses how blockchain can help supply chains to be greener, reducing waste and thus being more socially aware by providing insights and use cases on the subject.

This article focuses on detailing how collaborative business models can be implemented and potentially improved within permissioned blockchain, given how adaptable the technology is. Analogously, Chong et al. [5] paint the same view on a different angle, showing how business models can be adjusted, molded and re-imagined based on blockchain's capabilities. It emphasizes blockchain's impact on existing business models and, through careful and detailed review, analyzes business cases for different companies to come up with blockchain-specific business models. It proposes blockchain business models as a result of harmonizing business theory and practice, supplying its challenges and value creation for each case.

Collaborative businesses follow an overall business model that is generalized enough to fit broad categories of businesses. Di Ciccio et al. [10] draw attention to how modeldriven engineering can facilitate designing business processes using blockchain. Platforms that assist implementing Business Process Modeling Notation (BPMN) process diagrams into operational blockchain smart contracts are analysed. By doing so, it is possible to consolidate multiple companies relationships and operations with ease into a collaborative blockchain network with minimal business logic loss. This enables collaborating companies to achieve tighter bonds through blockchain.

The ideas presented in the studies aforementioned [5, 10] are designed to aid companies which already operate on a collaborative basis, such as supply chain and interoperating businesses. This article's efforts are to stress the positive outcomes of forming businesses alliances with companies that are not directly doing business together, but could well profit from doing so. Companies willing to join forces with diagonally operating businesses can extrapolate their responsibilities to reach previously untapped market and audience. Examples of said outcomes are further detailed in the upcoming sections.

\section{Business relationships}

In order to understand how blockchain can impact on business alliances, a few collaborative business models are introduced next to supply enough context for the reader to comprehend how elastic blockchain can be when addressing different business scenarios.

Due to blockchain's high levels of adaptability, it can benefit virtually every business relationships, whether those relationships are vertical or horizontal. 
- Vertical - In a vertical relationship setting businesses have often a supplier-customer relationship [21]. It means that businesses require products or services from other companies to deliver a final product to their customers.

- Horizontal - Horizontal relationships between businesses usually mean competing companies [21]. Horizontal relationships are related to common ground companies that cater to a similar target audience. This is where business alliances can be formed, acting together for the customers benefits as well as their own. This paper is most significant for horizontal relationships.

- Diagonal - Companies sometimes require services from other companies that do not operate in the same business. Diagonal relationships are relationships where companies are not directly related to other companies, but can impact them nevertheless.

Whether companies have horizontal, vertical or both kinds of relationship, the overall goal is the same: generate value for the target audience. When it comes to value creation, collaborative relationships can have different outcomes on organizations.

A sharing collaborative business model creates value by fusing matching capabilities in order to achieve a broader scale [9]. This is the case where companies with common clients (horizontal relationship) come together to improve customer satisfaction. It is worth noting that companies that share the same customers are not necessarily competitors. For instance, coffee shops and fine dinning restaurants both work on the food industry - they may share customers but they are not direct competitors.

Now, a complementary collaborative business model comes from companies working together to generate value that would be nonexistent should they work alone [9]. Combining different businesses with different purposes is where prime opportunities tend to arise. Although usually harder to integrate, complementary services have a more high-risk, high-reward feeling to it.

Other collaborative models exits, but given this paper's scope only the two aforementioned are relevant. In the following section collaborative blockchain use cases are described to provide a better picture of how collaborative businesses can take advantage of the blockchain technology.

\section{Collaborative blockchains}

In the following sub-sections, a couple of studies are reviewed to show how blockchain, specially permissioned blockchain, in real business scenarios reveals itself as a quite well-rounded solution for integrating businesses.

\subsection{Social responsibility use case}

The first scenario to be examined is the one described in Bedin et al.'s study [4] where, in an effort to drastically reduce energy disconnections and improve customer satisfaction, a local utility company aims to integrate and work in close partnership with assistance programs able to aid customers having difficulty in paying their electricity bills.

A contributing factor to disconnections being carried through is that clients are not aware of the existence of assistance programs they might be eligible for. In addition to that, the process of requesting assistance from one of the available programs is a lengthy and wearying endeavor. By the time the study was conducted, the process required paper copies of delayed bill warnings, in person visits to assistance programs through appointments and long request response wait times.

In this environment, the assistance program's aid is a prerequisite service for the electricity service to remain active. Figure 4 illustrates the comparison between the current scenario (4.a) in contrast to the scenario generated with blockchain (4.b). Permissioned blockchain can be adopted to act as a fast lane bridge between customers in need and assistance programs. In a blockchain network containing the energy providing company, its customers information and multiple partner assistance programs. On top of expediting the whole process, in a permissioned blockchain environment customers would become aware of which participating assistance programs they are eligible for through smart contracts and could act on it by requesting assistance. Requesting assistance through the network would result in significantly reduced request-response time considering that the process of sorting out which customer is qualified to which program would be automated in accordance with each program's eligibility criteria. Following that reasoning, the only remaining step on the entire process would be the assistance program approving or denying its support.

Assistance programs are positioned diagonally in regards to an energy provider, meaning that assistance programs are not in the business of providing electricity for their customers and the energy provider cannot provide financial support to their clients. On the same note, assistance programs often have a wider range of services other than just offering financial aid towards delayed electricity bills. That being said, those companies would collaborate in a complementary regime, where the utility company would direct customers in need to assistance programs. Such programs would respond by ensuring that the utility receives the correct payment for the services provided to their customers while granting uninterrupted energy services.

Permissioned blockchain allows for a swift integration between non-related business, removing technical barriers and speeding up relevant information exchange, making it a reliable fit for business relationships such as the one described. 


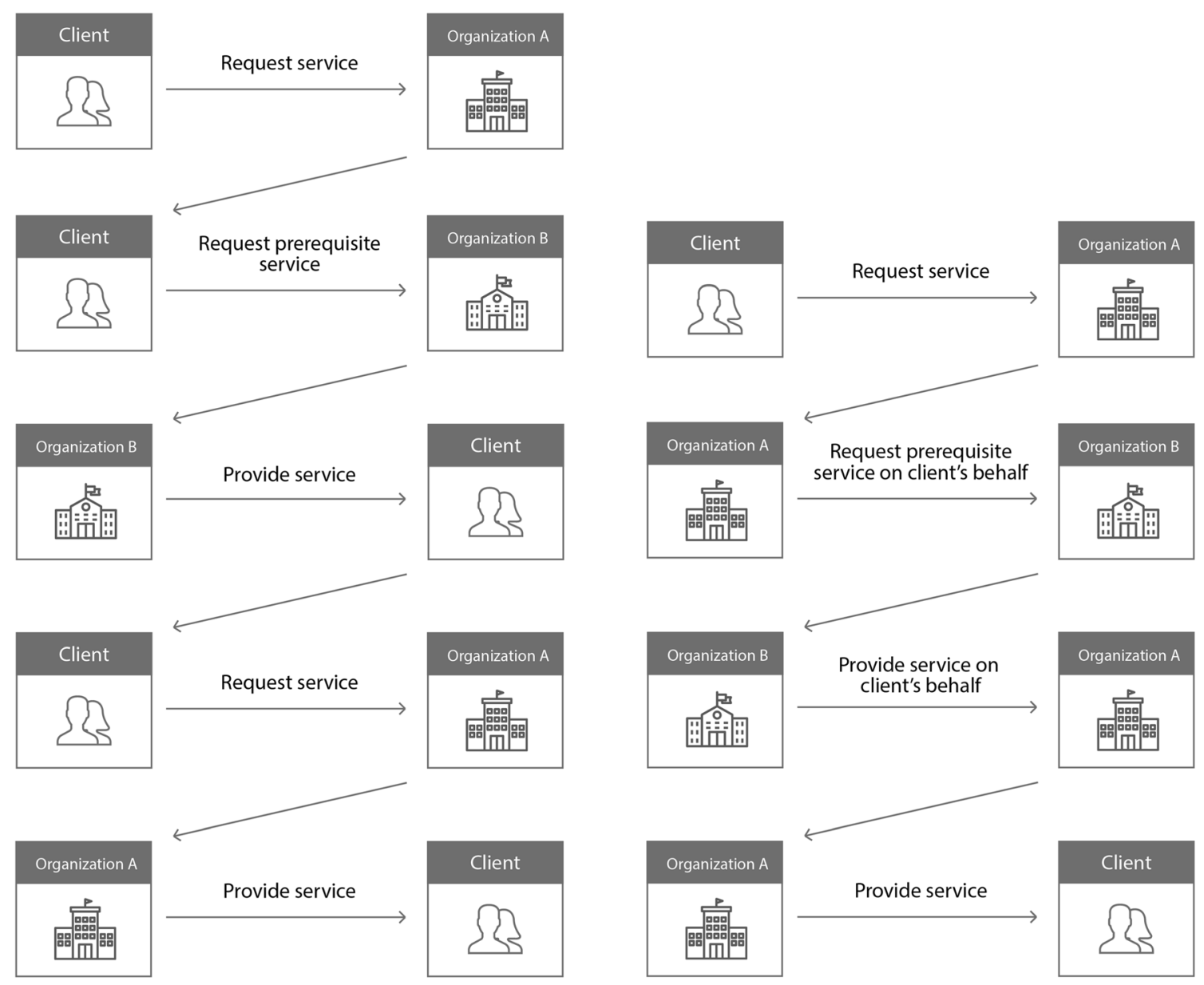

(a)

(b)

Fig. 4 Comparison of service delivery in a traditional setting (a) and through a permissioned blockchain (b)

\subsection{One-stop-shop use case}

In another business setting, companies that provide essential services often share the same body of clients. For example, all households requires gas, electricity, water and garbage collection at the very least. Additional nonessential services contracted for a household are often internet, phone and cable. All the services listed are provided to a single household, often enough assigned to the same person. In that regard, all the companies providing the services share the very same customer. Said customer has to individually contact every single company to obtain their services. From the client's perspective, the ideal situation would be to request all services required for its household through a single platform, in other words, a one-stop-shop. This would allow the customer's information to reach all contractors effortlessly in order to avoid filling out multiple very similar applications.
Blockchain accommodates the described one-stop-shop scenario smoothly. Figure 5 demonstrates how horizontally disposed companies can work together for their own interests as well as their client's. In a permissioned blockchain network, multiple essential and non-essential businesses can partner up to access shared customer information as required. The information can be appended on the blockchain by any of its participants, making it possible for the body companies in charge of providing services requested by said customer to do so without delay.

The one-stop-shop is a shining example of how horizontally related businesses can expand their reach when collaborating. With the introduction of an alliance, businesses can benefit of having clients introduced to their services that would otherwise be unknown to them. The one-stop-shop has a predictable value creation for their participants and users, with little to no downsides for the involved parties. 
Fig. 5 One-stop-shop use case. In stage 1 , the client yields its information to any of the service providers in a business blockchain. In stage 2, upon the client's request, any or every service provider within the network can now deliver their services to the client

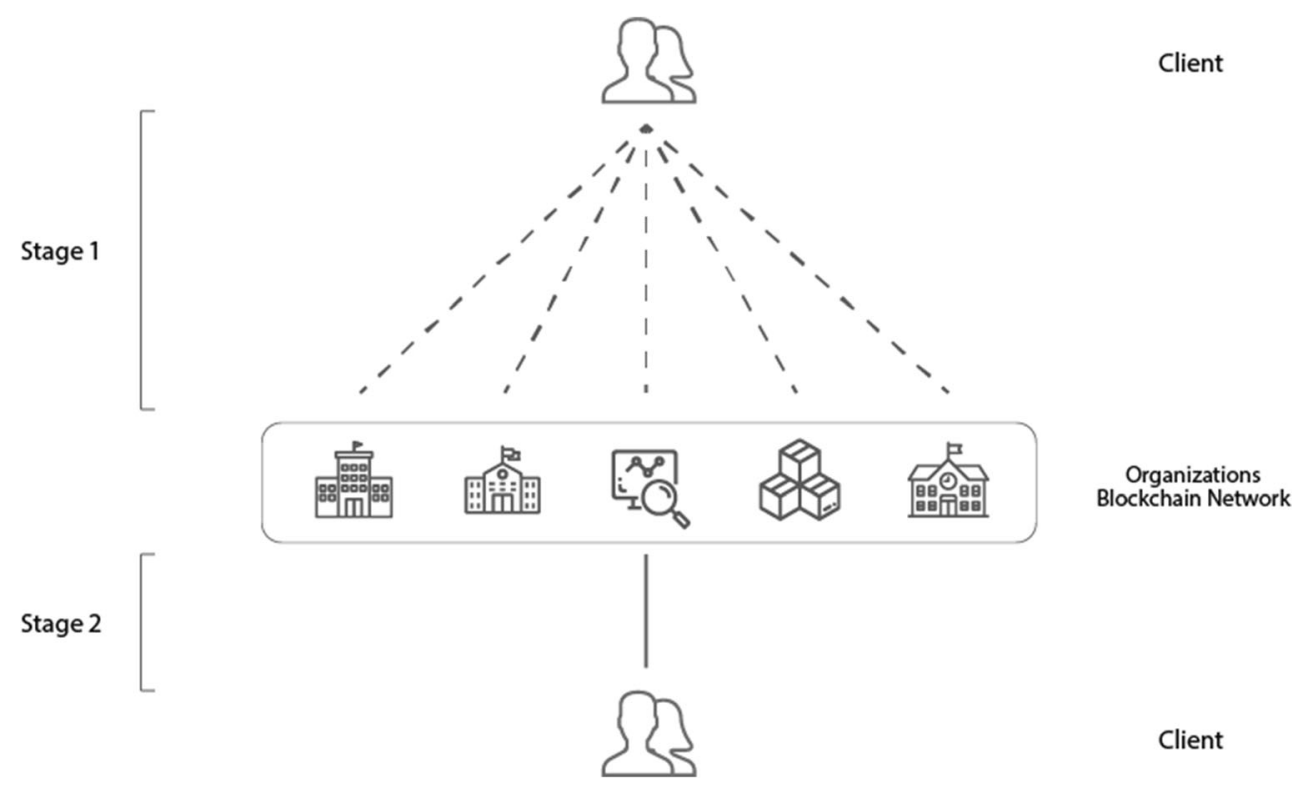

\section{Conclusions}

Blockchain is a multifaceted tool that can adapt to fit a great variety of purposes. Although most blockchain applications emphasize cryptocurrency and related financial technology, a plethora of other sectors remain fairly untouched by it. There is no reason to believe that organizations that work closely together would not benefit from operating within a permissioned blockchain setting provided that the transparency and security traits of blockchain can profoundly and positively impact the trust relationship between them.

Blockchain technologies can bring many value and benefits to companies, such as: secure transactions for collaborative business among them without costly billing systems changes; it streamlines business process for better, timely and lower cost customer experience; and ultimately can be a key element to enable smart city transactions to integrate enduser services in near real-time.

This study focused on demonstrating how companies can benefit from using blockchain to consolidate alliances with other businesses regardless of the business relation orientation among them. This allows companies to explore new audiences and possible clients that were once unreachable when doing isolated business. Blockchain, and specially permissioned blockchain, is flexible enough to accommodate differently related businesses. It can keep the technicalities of consolidating a network with multiple participants to a minimum, drastically reducing implementation downtime. The benefits described here seek to encourage companies to cooperate and prosper altogether ensuring that trust gaps among companies can be filled with the consensus protocol in blockchain networks.

Presented here were real-world scenario approaches illustrating the use of permissioned blockchain to collaborate with other businesses for their common benefit regardless of the business relationship between them. It highlights how working together with recognizable parties is paramount to better serve their clients However, businesses are often reluctant to cooperate because disclosing operational information can be seen as a threat rather than an opportunity for growth. This paper aims to foster the idea of using blockchain as a means to securely unveil information with other companies to mature and progress collectively, as well as introduce the idea of blockchain being an inviting and promising environment for businesses. Because a blockchain can centralize customer information, it can become a single means of access to such data, preventing customers of redundantly disclosing private information.

Finally, when it comes to business, use cases and history tends to dictate how to act on special situations. Business blockchains, although progressively more popular, still runs short of varied business circumstances. As a consequence of business blockchain growing notoriety that is expected to change. A systematic review and modernization of business models using blockchain is expected for industries to stay up to date on how to better work with it.

In terms of future work, the proof of concepts presented here could be implemented in a real business scenario to uncover non-trivial possibilities, allowing additional scenarios to be investigated in order to emphasize blockchain's ups and downsides even further. As value creation on diagonally related business is difficult to anticipate, numerous scenarios could be analysed on an empirical basis to ascertain yet hidden potential.

Acknowledgements This research has been partially supported by an OCE VIP II at Western University (VIP II - 31101). The authors would like to thank London Hydro for supplying industry knowledge used in this study. Additionally, the authors would like to thank Wander Queiroz for preliminary contributions and Shivam Dubey, Peter Esposto, Dane Sisinni and Yash Tolani for the one-stop-shop proof of concept project. 
Open Access This article is licensed under a Creative Commons Attribution 4.0 International License, which permits use, sharing, adaptation, distribution and reproduction in any medium or format, as long as you give appropriate credit to the original author(s) and the source, provide a link to the Creative Commons licence, and indicate if changes were made. The images or other third party material in this article are included in the article's Creative Commons licence, unless indicated otherwise in a credit line to the material. If material is not included in the article's Creative Commons licence and your intended use is not permitted by statutory regulation or exceeds the permitted use, you will need to obtain permission directly from the copyright holder. To view a copy of this licence, visit http://creativecommons.org/licenses/by/4.0/.

\section{References}

1. Allessie D, Sobolewski M, Vaccari L, Pignatelli F (2019) Blockchain for digital government. Publications Office of the European Union, Luxembourg

2. Andoni M, Robu V, Flynn D, Abram S, Geach D, Jenkins D, McCallum P, Peacock A (2019) Blockchain technology in the energy sector: a systematic review of challenges and opportunities. Renew Sust Energ Rev 100:143-174

3. Baliga A (2017) Understanding blockchain consensus models

4. Bedin ARC, Queiroz W, Capretz M, Mir S (2020) A blockchain approach to social responsibility. Data Information in Online Environments 319:1-15

5. Chong AYL, Lim ET, Hua X, Zheng S, Tan CW (2019) Business on chain: a comparative case study of five blockchain-inspired business models. J Assoc Inf Syst 20(9):9

6. Christidis K, Devetsikiotis M (2016) Blockchains and smart contracts for the internet of things. Ieee Access 4:2292-2303

7. Chua PHT, Li Y, He W (2019) Adopting hyperledger fabric blockchain for epcglobal network. In: 2019 IEEE International Conference on RFID (RFID), IEEE, pp 1-8

8. Crosby M, Pattanayak P, Verma S, Kalyanaraman V et al (2016) Blockchain technology: beyond bitcoin. Applied Innovation 2(610):71

9. de Man AP, Luvison D (2019) Collaborative business models: aligning and operationalizing alliances. Business Horizons 62(4): 473-482

10. Di Ciccio C, Cecconi A, Dumas M, Garća-Bañuelos L, L'opezPintado O, Lu Q, Mendling J, Ponomarev A, Tran AB, Weber I (2019) Blockchain support for collaborative business processes. Informatik Spektrum 42(3):182-190

11. Dorri A, Kanhere SS, Jurdak R, Gauravaram P (2017) Blockchain for iot security and privacy: The case study of a smart home. In: 2017 IEEE international conference on pervasive computing and communications workshops (PerCom workshops), IEEE, pp 618623

12. Hebert C, Di Cerbo F (2019) Secure blockchain in the enterprise: a methodology. Pervasive and Mobile Computing pp 10-38
13. Iansiti M, Lakhani KR (2017) The truth about blockchain. Harvard Business Review 95(1):118-127

14. Kirillov D, Korkhov V, Petrunin V, Makarov M, Khamitov IM, Dostov V (2019) Implementation of an e-voting scheme using hyperledger fabric permissioned blockchain. In: International Conference on Computational Science and Its Applications, Springer, pp. 509-521

15. Kouhizadeh M, Sarkis J (2018) Blockchain practices, potentials, and perspectives in greening supply chains. Sustainability 10(10): 3652

16. Lev-Ari K, Spiegelman A, Keidar I, Malkhi D (2019) Fairledger: a fair blockchain protocol for financial institutions. arXiv preprint arXiv: 190603819

17. Li J (2018) Public philanthropy logistics platform based on blockchain technology for social welfare maximization. In: 2018 8th international conference on logistics, Informatics and Service Sciences (LISS), IEEE

18. Liang X, Zhao J, Shetty S, Liu J, Li D (2017) Integrating blockchain for data sharing and collaboration in mobile healthcare applications. In: 2017 IEEE 28th Annual International Symposium on Personal, Indoor, and Mobile Radio Communications (PIMRC), IEEE, pp 15

19. Liu KH, Chang SF, Huang WH, Lu IC (2019) The framework of the integration of carbon footprint and blockchain: using blockchain as a carbon emission management tool. In: Technologies and Ecoinnovation towards Sustainability I, Springer, pp. 15-22

20. McGhin T, Choo KKR, Liu CZ, He D (2019) Blockchain in healthcare applications: research challenges and opportunities. J Netw Comput Appl 135:62-75

21. Meador AL, Church PH, Rayburn LG (1996) Development of prediction models for horizontal and vertical mergers. Journal of financial and strategic decisions 9(1):11-23

22. Miraz MH, Ali M (2018) Applications of blockchain technology beyond cryptocurrency. arXiv preprint arXiv: 180103528

23. Mukkamala RR, Vatrapu R, Ray PK, Sengupta G, Halder S (2018) Blockchain for social business: principles and applications. IEEE Engineering Management Review 46(4):94-99

24. Nakamoto S (2019) Bitcoin: a peer-to-peer electronic cash system. Tech. rep., Manubot

25. Ølnes S, Ubacht J, Janssen M (2017) Blockchain in government: benefits and implications of distributed ledger technology for information sharing

26. Scott B (2016) How can cryptocurrency and blockchain technology play a role in building social and solidarity finance? Tech. rep., UNRISD Working Paper

27. Xia Q, Sifah EB, Asamoah KO, Gao J, Du X, Guizani M (2017) MeDshare: trust-less medical data sharing among cloud service providers via blockchain. IEEE Access 5:14757-14767

Publisher's note Springer Nature remains neutral with regard to jurisdictional claims in published maps and institutional affiliations. 